SESSION 2. THE PHYSICS OF THE SYMBIOTIC PHENOMENON

"Stars regain equilibrium after coughing" Mario Livio 


\title{
Ionization models of symbiotic stars
}

\author{
H. Nussbaumer \\ Institute of Astronomy \\ ETH Zentrum \\ 8092 Zürich (Switzerland)
}

SUMMARY. A model of a symbiotic system is outlined. We present evidence that the main ionization mechanism responsible for the symbiotic nebula is radiative and not collisional. For calculating fractional ionization throughout the emission region we take either a blackbody source or an accreting system. If the ionizing radiation is due to accretion, the boundary layer between accreting star and disc provides the bulk of the ionizing photons. The possibility for distinguishing between different ionization sources are briefly discussed. $\mathrm{C} / \mathrm{N}$ and $\mathrm{O} / \mathrm{N}$ abundance ratios, calculated with the outlined ionization model places $\mathrm{HM}$ Sge, RS Oph, and T CrB among the novae and not among the symbiotic stars.

\section{Introduction}

When the first IAU colloquium on symbiotic stars was held at the Haute Provence Observatory in August 1981, the ultraviolet observatory IUE (International Ultraviolet Explorer) had just about finished three years of operation. Already the first results made it obvious that for symbiotic stars IUE had indeed opened an essential part of the spectral range, containing vital information for deriving ionization models. The new observations stimulated a wave of research in symbiotic stars, and the results presented at the 1981 colloquium were impressive. Concerning ionization models, it is now my task to present what has happened in the six years since 1981, and to outline what is required in the near and medium future. - Although the sudden glamorous impact of IUE is certainly responsible for the revival of interest in symbiotic systems (e.g. Nussbaumer and Stencel 1987), the progress in our knowledge would be unthinkable without the infrared (e.g. Feast et al.1983) and radio observations.

When IUE went up, UV spectroscopy of symbiotic stars was taken up without need of much additional line-identifications. The list published by Penston et al.(1983, but worked on and circulated much earlier) on lines identified in RR Tel, was a summary of knowledge that had been accumulated since the times when spectroscopy of the solar transition region was a new field. It was also helped by studies of planetary nebulae and QSO. 


\section{Spectroscopy at the 1981 colloquium on symbiotic stars}

Are all symbiotic stars binary systems? Neither infrared nor visual, nor UV observations on their own can provide a general answer to that question: indeed on the basis of each of those observations one can certainly construct single star models for many symbiotic systems. The planetary nebula type model, as published for example by Nussbaumer and Schild (1981) in order to explain the UV spectrum of V $1016 \mathrm{Cyg}$, did not need a double star system to match model and observation. And only rarely do periodic wavelengthshifts prove the existence of a double star system, as in the case of HBV 475. The proof of the double star nature of an object like V 1016 Cyg comes mainly from the combination of infrared and UV observations.

The main results of the first phase of UV studies of symbiotic stars was to show that ionization is done by radiation and not by collisions. This then implies the presence of a hot radiation source. How does one prove that symbiotic emission regions are radiatively ionized? We show that the electron temperature, $T_{e}$, responsible for the collisional excitation of observed spectral lines, is too low to produce the observed ionization stages by collisional ionization. Suitable lines for a determination of $T_{e}$ are scarce. C III and Si III can be used. C III] $\lambda 1909$ corresponds to the transition $2 \mathrm{~s}^{2}{ }^{1} \mathrm{~S}_{0}-2 \mathrm{~s} 2 \mathrm{p}{ }^{3} \mathrm{P}_{1}^{o}$. We compare it to the strength of the multiplet $2 \mathrm{~s} 2 \mathrm{p}{ }^{3} \mathrm{P}^{o}-2 \mathrm{p}^{2}{ }^{3} \mathrm{P}$ at $\lambda 1176$. In a collisionally ionized gas C III is seen if $T_{e} \approx 50000 \mathrm{~K}$. But at that temperature, and $\mathrm{N}_{e}>10^{6} \mathrm{~cm}^{-3}$, the C III $\lambda 1176$ multiplet should have about one third of the strength of $\lambda 1909$ (Nussbaumer and Schild, 1979). However, in symbiotic stars C III $\lambda 1176$ does not appear with that strength. We may therefore conclude that $T_{e}<50000 \mathrm{~K}$. We now turn to Si III $3 \mathrm{~s}^{2}{ }^{1} \mathrm{~S}_{0}-3 \mathrm{~s} 3 \mathrm{p}{ }^{3} \mathrm{P}_{1}^{o}$ $\lambda 1892$ and the multiplet $3 \mathrm{~s}^{2}{ }^{1} \mathrm{~S}_{0}-3 \mathrm{~s} 3 \mathrm{p}{ }^{1} \mathrm{P}_{1}^{o}$ at $\lambda 1206.5$. With the help of these lines Hayes and Nussbaumer (1986) have determined $T_{e}$ in RR Tel, they find $T_{e}=(1.3 \pm 0.2) \times 10^{4}$ $K$. This rules out collisional ionization for the main emission regions of the nebular lines. It would not rule out that some of the lines - from either higher or lower ionization stages - could originate from a collisionally ionized region. However, the spectral evidence gives no indication for such conditions, with, perhaps, the exception of X-ray observations. Evidently, extension of the observing range down to $912 \AA$, where many of the resonance lines appear, would greatly facilitate $T_{e}$-determinations.

\section{Shift on emphasis}

As the binary (multiple?) nature of symbiotics has again become the generally accepted opinion, research in symbiotic stars has been shifting to the physical processes taking place within those systems. The fashion for accretion discs has also caught up with symbiotic stars. Models are at present mainly concerned with the following three components:

1) a red giant or supergiant,

2) a hot radiation source,

3) a nebula.

Depending on the authors, there may be coexistence or interaction. Because of geometry and chemical composition the origin of the nebula matters: is it a remnant of what now is the hot radiation source, or is it the stellar wind from the cool star? Nussbaumer et al.(1987) have compared $\mathrm{C} / \mathrm{N}$ and $\mathrm{O} / \mathrm{N}$ abundance ratios of red giants and supergiants, carbon stars, planetary nebulae, novae, and symbiotic stars. In Fig.1 I show some of the 
results. The symbiotic stars lie in the region of the M-giants, and are clearly separated from novae. The authors interprete this result as proof that symbiotic nebulae are composed of the mass lost by the red giant, and that the nebulae are not the product of nova-like nuclear burning, as would be expected in nova-like symbiotic models (e.g. Paczynski and Rudak, 1980); planetary nebulae occupy a yet different field of the plot. It is worth adding that RS Oph and T CrB have been counted as novae.

Figure 1. Relative abundances $\mathrm{O} / \mathrm{N}$ plotted against the relative abundances $\mathrm{C} / \mathrm{N}$ for red giants, carbon stars, novae, and symbiotic stars (Nussbaumer et al.1987) The relative abundances are given on a logarithmic scale. The symbols stand for the sun: $\odot$, Gand $\mathrm{K}$-giants: $\mathrm{x}, \mathrm{M}$-giants: + , novae: $\circ$, carbon stars: $\bullet$, symbiotic stars: *. The symbiotic star among the novae is HM Sge.

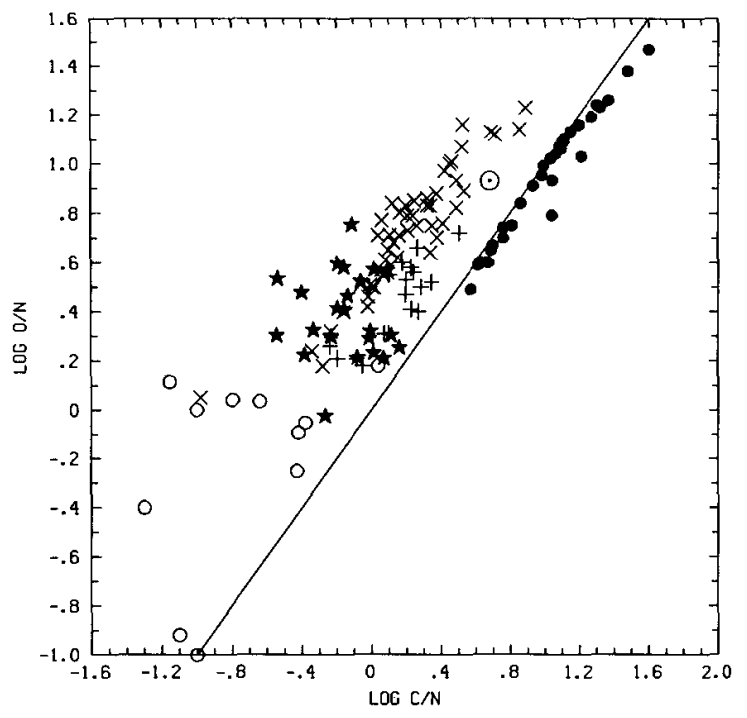

One cannot talk about ionization models without specifying a model of the symbiotic system as a whole. I shall proceed on the basis of the symbiotic model of Nussbaumer and Vogel (1987). They suggest that the emission line region observed in symbiotic stars is provided by the wind of the red giant, and that the onset of such a wind may explain the outbreak of symbiotic stars. If that theory is correct, the wind from the cool star is an essential feature in symbiotics. As a source of hot radiation the following candidates are in the running:

(1) normal hot star with blackbody or non-blackbody radiation field

(2) accretion disc and its non-blackbody radiation field.

Because of their basic differences the nature of the source of radiation has - independent of its importance for the ionization structure - become itself a debated object of investigation.

We then assume that the main components and features of a symbiotic system can be described in the following way:

The double star pair consists of a mass-losing red giant or supergiant, and a dwarf, subdwarf or white dwarf, which emits the radiation ionizing the nebula. The dwarf-type star may form an accreting system. The line and continuum radiation at visual, $U V$ and radio wavelengths is mainly due to the nebular emission and to hot stellar or accretion system contribution. The red part of the visual, and the infrared radiation is emitted by the cool mass-loosing star and by dust in its wind. A fraction of the mass lost by the cool star may be captured by the companion, either by accretion from a wind or through Roche-lobe 
overflow. The accreted mass may form a disc around the companion. In the latter case the accretion disc could be the main source for the hot radiation field.

\section{Ionization models}

\subsection{General remarks.}

In the ionization model we assume an equilibrium situation. Thus for each volume element the number of ionizations is equal to the number of recombinations:

$$
N\left(X^{+i}\right) \rightleftharpoons N\left(X^{+i+1}\right) .
$$

We consider that equilibrium is established by the following processes:

radiative ionization and two-body recombination (including dielectronic recombina-

tion)

collisional ionization and three-body recombination

charge exchange

The most important of these processes are: radiative ionization with its inverse - twobody recombination - and charge exchange. The radiation field is composed of the stellar radiation, $J_{\nu}^{*}$, and the diffuse field, $J_{\nu}^{d}$, created in the nebula:

$$
J_{\nu}=J_{\nu}^{*}+J_{\nu}^{d}
$$

For a source radiating spherically symmetrically, we have

$$
4 \pi J_{\nu}^{*}(r)=\pi F_{\nu}^{*}\left(R^{*}\right)\left(\frac{R^{*}}{r}\right)^{2} e^{-\tau_{\nu}(r)}
$$

where the optical depth $\tau$ is

$$
\tau_{\nu}(r)=\sum_{X+m} \int_{R^{*}}^{r} N\left(X^{+m}\right) a_{\nu}\left(X^{+m}\right) d s .
$$

In principle this sum should be extended over all the continuum and line absorptions active at frequency $\nu$. The difficulty lies in properly treating $J^{d}$. Apart from strong spectral lines, which can have an ionizing effect, $J^{d}$ is mainly created by recombination within the nebula. At a given position $\vec{r}$ in the nebula we should add the contribution to $J^{d}$ from all other positions $\vec{r}$, attenuated by optical depth. However, recombination is usually treated in a much simpler fashion, I shall briefly outline how the ionizing field $J$ at a position $\vec{r}$ is obtained in our approach. We follow the ionizing paths of the stellar radiation in straight lines, starting from the ionizing star S2. Along this path $J$ is the result of the attenuated stellar radiation (3) and the diffuse radiation accumulated along the path between $\mathrm{S} 2$ and $\vec{r}$. At the position $\vec{r}$ we only regard the recombination radiation arising in a small volume around position $\vec{r}$. We assume that those photons which are capable of ionizing $\mathrm{H}$ or $\mathrm{He}$ will do so, the others are added to $J^{d}$; this includes line emission due to collisional excitation.

Fig. 2 shows the model configuration which will be employed in the following chapters. A cool mass-losing star, S1, is accompanied by a source of hot radiation, $\mathrm{S} 2$. S2 may be stellar-like, in which case it is assumed to radiate as a blackbody. It may be also be an 
accreting system, in which case the accretion-disc and the boundary layer are supposed to be the source of the ionizing radiation. We assume the mass-loss of the cool star to be given by

$$
\dot{M}=4 \pi r^{2} \mu m_{H} N(r) v_{\infty}\left(1-\frac{R}{r}\right) .
$$

In the examples shown here, the origin of the stellar wind lies at $R=50 R_{\odot}, v_{\infty}=80$ $\mathrm{km} / \mathrm{s}$, and for the mass-loss we set $M=3.5 \cdot 10^{-6} M_{\odot} / y r$. For the nebula we thus have a $\approx 1 / r^{2}$ density distribution, with density decreasing towards the source of ionizing radiation. $\mathrm{S} 1$ and $\mathrm{S} 2$ are separated by $5 \cdot 10^{13} \mathrm{~cm}$. The model of Figure 2 was originally proposed by Nussbaumer and Schmutz (1983) and further developped by Nussbaumer and Vogel (1987).

Figure 2. Model of a symbiotic object. A fraction of the wind of a cool, mass-loosing star is ionized by a hot radiation source. hot object

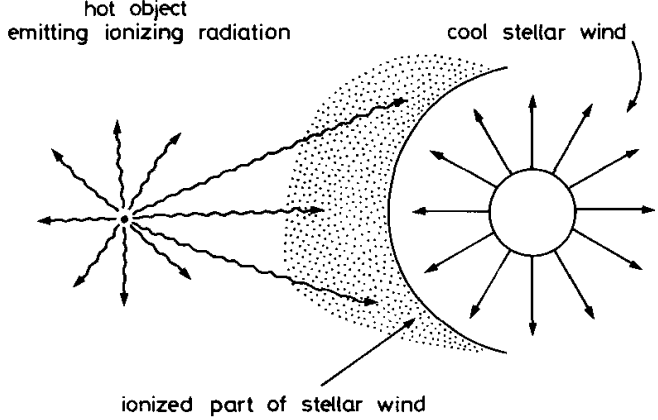

\subsection{Black-body radiation sources.}

The observed ionization stages in symbiotics mostly indicate high $T^{*}$, I shall assume $T^{*}=$ $150000 \mathrm{~K}$ and $R^{*}=9 \cdot 10^{8} \mathrm{~cm}$. - It was pointed out by Hayes and Nussbaumer (1986) that with the presently observable spectra we cannot determine $T^{*}$ with high accuracy if $T^{*} \gtrsim 150000 \mathrm{~K}$. - We first compare the resulting ionization structure with that from a nebula of constant density $N(\mathrm{H})$. The density in the constant-density model was chosen to give the same Stroemgren-radius on the S1-S2 path as the $1 / r^{2}$-model, it resulted in $N(\mathrm{H})=8.53 \cdot 10^{8} \mathrm{~cm}^{-3} ; N(\mathrm{H})=N\left(\mathrm{H}^{+}\right)+N\left(\mathrm{H}^{0}\right)$. Results along the radiation path $\mathrm{S} 2 \rightarrow$ $\mathrm{S} 1$ are given in Figures $3-5$ for He, C, N, O, Si, and Fe. From Fig. 3 and Fig. 4 we see that in the $1 / r^{2}$ density distribution the higher ionization stages approach the cool mass-loosing star more closely than in the constant density model. However, the two density distributions result in qualitatively similar ionization structures. This is due to the high mass-loss rate; for $\dot{M}=3.5 \cdot 10^{-6} M_{\odot} / y r$ the nebula resulting from our configuration appears almost as one of constant density. According to the ionization state of He we distinguish between the $\mathrm{He}^{+2}$ and the $\mathrm{He}^{+}$zones. (For $T^{*} \gtrsim 40000 \mathrm{~K}$ the $\mathrm{He}^{0}$-region coincides with the $\mathrm{H}^{0}$ region.) As the prominent $\mathrm{He}$-lines in symbiotic systems are recombination lines, the two zones correspond to the He II and the He I emission regions. The $\mathrm{He}^{+}$zone corresponds to the region where $\mathrm{O}$ III and Fe IV are emitted, it is also the region where $\mathrm{C}$ III and N III are emitted. There is practically no emission of $\mathrm{Fe} \mathrm{V}$ and only a narrow Fe VI emission region, corresponding to the O IV region. Fe VII and $O$ V, and Fe VIII and O VI are emitted in about the same regions respectively. In the innermost part of the $\mathrm{He}^{+2}$ region oxygen exists as $\mathrm{He}-$ like $\mathrm{O}^{+6}$, whereas $\mathrm{Fe}$ emits mainly $\mathrm{Fe} \mathrm{IX}$, but also some $\mathrm{Fe} \mathrm{X}$ and $\mathrm{Fe} \mathrm{XI}$ in tiny, hardly observable amounts. 

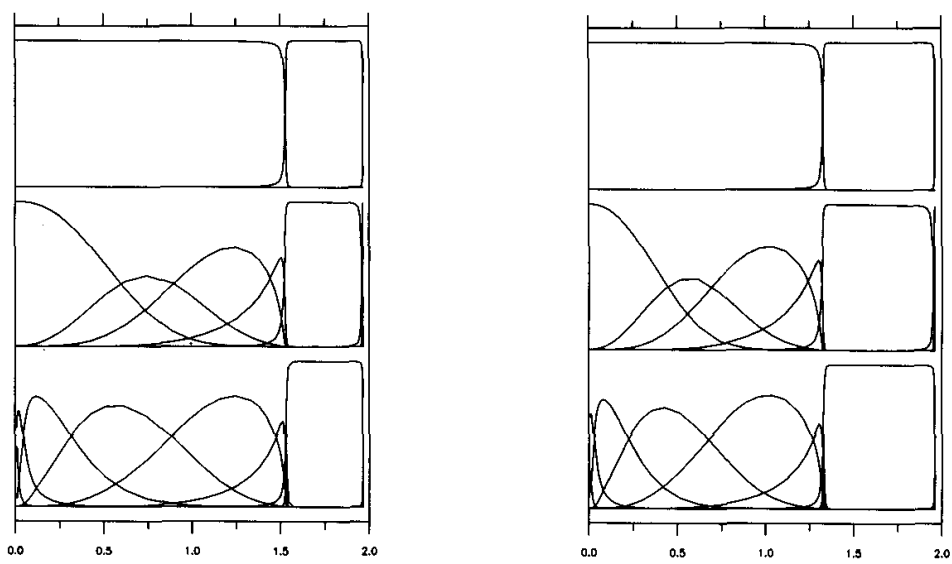

Figure 3 (left). The ionization structure of helium, oxygen, and iron (from top to bottom) calculated with a $N(r) \sim 1 / r^{2}$ density distribution. Given is the fractional ionization $N\left(\mathrm{X}^{+m}\right) / N(\mathrm{X})$. The hot radiation source, with $T^{*}=1.5 \cdot 10^{5} \mathrm{~K}, R^{*}=9 \cdot 10^{8} \mathrm{~cm}$, is placed at $r=0$, distances from that point are given in units of $10^{13} \mathrm{~cm}$. The cool mass-loosing star is separated by a distance $p=5 \cdot 10^{13} \mathrm{~cm}$ from the hot star. The resulting nebula corresponds to the closed model in Fig.1 of Nussbaumer and Vogel (1987). The ionization stages in order of their maxima with growing distance from the radiation source are for He: $\mathrm{He}^{+2}, \mathrm{He}^{+}$, for $\mathrm{O}: \mathrm{O}^{+6}, \mathrm{O}^{+5}, \mathrm{O}^{+4}, \mathrm{O}^{+3}, \mathrm{O}^{+2}, \mathrm{O}^{+}$(very narrow), for $\mathrm{Fe}: \mathrm{Fe}^{+10}, \mathrm{Fe}^{+9}$, $\mathrm{Fe}^{+8}, \mathrm{Fe}^{+7}, \mathrm{Fe}^{+6}, \mathrm{Fe}^{+5}, \mathrm{Fe}^{+4}$ (practically not visible), $\mathrm{Fe}^{+3}$, and as very narrow peaks at the boundary appear $\mathrm{Fe}^{+2}$ and $\mathrm{Fe}^{+}$.

Figure 4 (right). The ionization structure of helium, oxygen, and iron, as in Fig. 3 but calculated with $N(r)=$ constant $=4.03 \times 10^{8} \mathrm{~cm}^{-3}$.

Figure 5. The ionization structure of carbon, nitrogen, and silicon, obtained with the same parameters as the curves of Fig. 4. The ionization stages in order of their maxima with growing distance from the radiation source are for $\mathrm{C}: \mathrm{C}^{+4}, \mathrm{C}^{+3}, \mathrm{C}^{+2}, \mathrm{C}^{+}$(peak at the boundary); for $\mathrm{N}: \mathrm{N}^{+5}, \mathrm{~N}^{+4}, \mathrm{~N}^{+3}, \mathrm{~N}^{+2}, \mathrm{~N}^{+}$ (peak at the boundary); for $\mathrm{Si}: \mathrm{Si}^{+7}, \mathrm{Si}^{+6}$, $\mathrm{Si}^{+5}, \mathrm{Si}^{+4}$ (containing most of $\mathrm{Si}$ ), $\mathrm{Si}^{+3}, \mathrm{Si}^{+2}$ and $\mathrm{Si}^{+}$(as very narrow peak at the boundary).

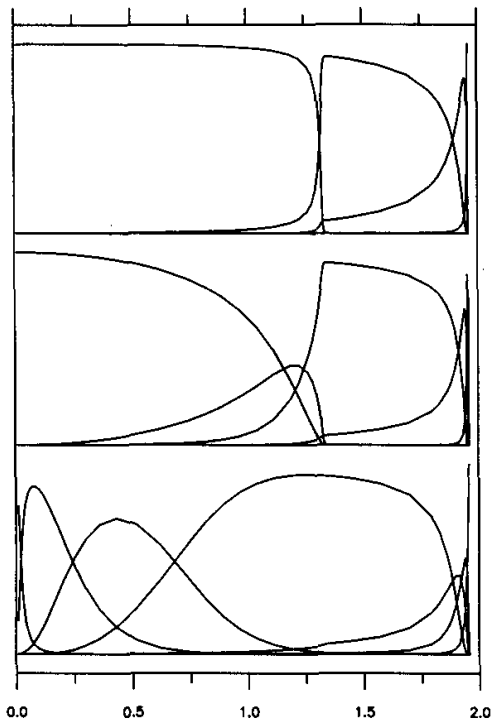


The neat division between $\mathrm{O}^{+2}$ and its neighbours, as well as between $\mathrm{Fe}^{+3}$ and other ionization stages of $\mathrm{Fe}$, is due to their respective ionization potentials being just slightly higher - less than $1 \mathrm{eV}$ - than that of $\mathrm{He}^{+}$. As the ionization potentials of $\mathrm{C}^{+2}$ and $\mathrm{N}^{+2}$ are similar, we find also a fairly neat separation in those elements. Within the $\mathrm{He}^{+}$region $\mathrm{C}, \mathrm{N}, \mathrm{O}$, and also $\mathrm{Fe}$ are represented by one or two ionization stages. This is not a general feature, $\mathrm{Si}$ being an example to the contrary. The $\mathrm{Si}$ ions $\mathrm{Si}^{+}, \mathrm{Si}^{+2}, \mathrm{Si}^{+3}$ have ionization potentials of 16,33 , and $45 \mathrm{eV}$. We see that in the $\mathrm{He}^{+}$zone $\mathrm{C}$ is represented by $\mathrm{C}$ III and $\mathrm{C}$ IV, $\mathrm{N}$ by $\mathrm{N}$ III and $\mathrm{N}$ IV, $\mathrm{O}$ by $\mathrm{O}$ III, Fe by Fe IV, however, Si by Si II, Si III, Si IV and Si V. As all four corresponding ionization stages are present, there is a rather large uncertainty about the fractional abundance of each individual stage. This concerns in particular $\mathrm{Si}$ III, as $\mathrm{Si}^{+2}$ is less abundant than either $\mathrm{Si}^{+3}$ or $\mathrm{Si}^{+4}$. The temptation, to use the prominent Si III $\lambda 1892$ line as representative for $\mathrm{Si}$ is therefore dangerous.

As long as densities are sufficiently low to render collisional de-excitation ineffective, differences between the two density distributions do not effect relative line strengths. The reason is the relation between the radiation source $\left(T^{*}, R^{*}\right)$ and the mass-loss. The present model, with $\dot{M}=3.5 \cdot 10^{-6} M_{\odot} / y r$ results in a closed nebula (but see also the figures in Nussbaumer and Vogel, 1987). It is of interest to compare relative fluxes of ions with ionization energies above and below the $\mathrm{He}^{+}$ionization energy. Thus $\mathrm{O} \mathrm{V}$ and $\mathrm{O}$ IV originate mainly in the $\mathrm{He}^{+2}$ region, whereas $\mathrm{O}$ III originates in the $\mathrm{He}^{+}$region. As an example we compare the ratios O III $\lambda 1660 / \mathrm{O}$ IV $\lambda 1400 / \mathrm{O} V \lambda 630$ for of the total nebular fluxes for the two models. Normalising the multiplet O III $\lambda 1663$ in the $N(r) \sim 1 / r^{2}$ model to 100 , we find:

O V $\lambda 630 /$ O IV $\lambda 1400 / O$ III $\lambda 1663=6 / 39 / 100$ for the $N(r) \sim 1 / r^{2}$ model and

O V $\lambda 630 /$ O IV $\lambda 1400 /$ O III $\lambda 1663=7 / 37 / 102$ for the $N(r)=$ constant model.

\subsection{Accretion discs.}

We shall now turn to accretion discs. The luminosity in an accretion disc is obtained through conversion of kinetic into radiative energy when particles on their orbits drift closer to the accreting star. Our model for the emitted spectrum is mainly based on the work of Shakura and Sunyaev (1973), Lynden-Bell and Pringle (1974), and Pringle (1981 and ref.cit.). At any given time the particles are supposed to be on Keplerian orbits, except for the inner boundary where the Keplerian orbits have been stopped. The inward drifting is due to viscosity. According to the virial theorem, half of the energy available to a particle is radiated away within the trajectory through the disc, and the other half on the arrival at the boundary layer. The main uncertainties are viscosity and the structure of the boundary layer.

For calculating the flux, $F_{\nu}^{d i s c}$, emitted by the accretion disc, a temperature $T(r)$ can be defined at any point in the accretion disc. This function has the form

$$
T(x)=T_{*}\left(x^{-3}-x^{-\frac{7}{2}}\right)^{\frac{1}{4}}
$$

where

$$
x=\frac{r}{R_{*}}, \quad \text { and } \quad T_{*}=\left(\frac{3 G M \dot{M}}{8 \pi \sigma R_{*}^{3}}\right)^{1 / 4} .
$$

$R_{*}$ is the radius of the boundary layer. We further find $T_{\max }=0.488 \times T_{*}$. In Figure 6 we show $\mathrm{T}(\mathrm{x})$ for $1 \leq x \leq 10$. 
Lynden-Bell and Pringle (1974) and Pringle (1977) have investigated the properties of the inner boundary. The temperature, $T^{b l}$, calculated for the boundary layer depends on the physical assumptions concerning the structure of the boundary layer. The work of LyndenBell and Pringle gives a choice of three temperatures. Lynden-Bell und Pringle (1974) calculate the thickness of the boundary layer from the Navier-Stokes relations, neglecting pressure. Pringle (1977) includes pressure. A different $\mathrm{T}^{b l}$ results when opacity due to electron scattering is included. All three results have the form

$$
T^{b l} \sim(\dot{M})^{\alpha}(M)^{\beta}\left(\frac{1}{R_{*}}\right)^{\gamma}
$$

The values for $\alpha$ and $\beta$ vary between $2 / 9$ and $4 / 7$, those for $\gamma$ between $3 / 4$ and $18 / 19$. The total flux emitted from the accretion disc and the boundary layer is

$$
F_{\nu}^{t o t}=F_{\nu}^{d i s c}+F_{\nu}^{b l}
$$

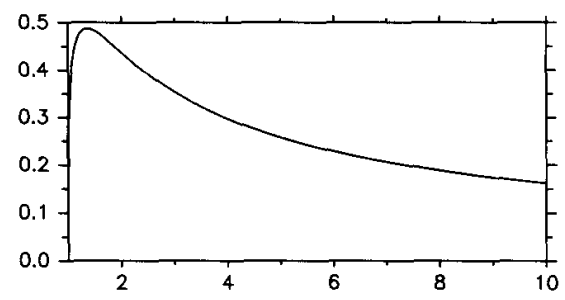

Figure 6. The radiation temperature of the accretion disc as a function of the distance from the inner boundary of radius $R_{*}$. The temperature is given in units of $T_{*}$, the distance is given as $x=r / R_{*}$.

The separation of the accretion system in disc and boundary layer is artificial. This is obvious from the drop in $T(x)$ to zero for $x=1$, as shown in Fig.6. The work of Regev (1983) is an example for the attempt to match disc and boundary layer.

Vogel (1988) will report in some detail on the differences expected between ionization from accretion discs or blackbodies. In the next section I shall summarily mention his main results.

\section{Accretion}

Is accretion an essential feature in symbiotic systems? Based on the work of Livio et al.(1986), who place severe limits on the accretion cross section, Nussbaumer and Vogel (1987) conclude that if the hot companion moves in a spherically symmetrical wind emitted by the cool component, there is little likelyhood of accretion disc formation in symbiotic systems. The case is different, if the giant fills the Roche-lobe.

Kenyon and Webbink (1984) have set themselves the task of finding the most pronounced observable spectral differences longwards of $1000 \AA$ among the various ionization models, including accretion systems. However, to avoid dealing with the many free parameters of the nebular emission, they restrict their efforts to the continuum flux from the 
stars or discs. They find the following configurations to be the most likely models for hot sources:

(a) Main sequence stars accreting $\approx 10^{-5} M_{\odot} / y r$. Such large rates require the giants of these systems (E Aud, CI Cyg, YY Her, AR Pav) to fill their Roche lobes. The nature of the giants could not be properly determined.

(b) The rest of the 15 systems observed they explain by the presence of a hot stellar source $25000 \mathrm{~K}<T^{*}<120000 \mathrm{~K}$; but they are subdwarfs rather than white dwarfs.

They think that the two types are different in their outburst mechanism. The main sequence accretor outbursts are thought to be dwarf-nova-type outbursts, whereas the hot star outbursts are thought to be rather of the nova-type outburst.

But can we confidently distinguish, from presently available spectral features, whether the source of hot radiation is a blackbody stellar object or an accretion disc? Vogel (1988) discusses the details of this problem. The results he found do not agree on all points with earlier investigation by Kenyon and Webbink (1984). Vogel finds that on the basis of emission line spectroscopy it is almost impossible to distinguish between a blackbody source and a main sequence accretor. Uncertainties in the theoretical treatment of the boundary layer are partly responsible for this state.

Figure 7. The ionization structure of hydrogen, helium, carbon, and iron, obtained in the radiation field of an accreting white dwarf. The ionization stages in order of their maxima with growing distance from the radiation source are for $\mathrm{H}: \mathrm{H}^{+}, \mathrm{H}^{0}$, for $\mathrm{He}: \mathrm{He}^{+2}, \mathrm{He}^{+}$, $\mathrm{He}^{0}$, for $\mathrm{C}: \mathrm{C}^{+6}, \mathrm{C}^{+5}, \mathrm{C}^{+4}, \mathrm{C}^{+3}, \mathrm{C}^{+2}, \mathrm{C}^{+}$, for $\mathrm{Fe}: \mathrm{Fe}^{+15}$ and higher, then follow $\mathrm{Fe}^{+14}$ and lower stages, reaching a peak for $\mathrm{Fe}^{+3}$. For the region covered by $\mathrm{H}^{0}$ see text. Distances are given in units of $10^{15} \mathrm{~cm}$.

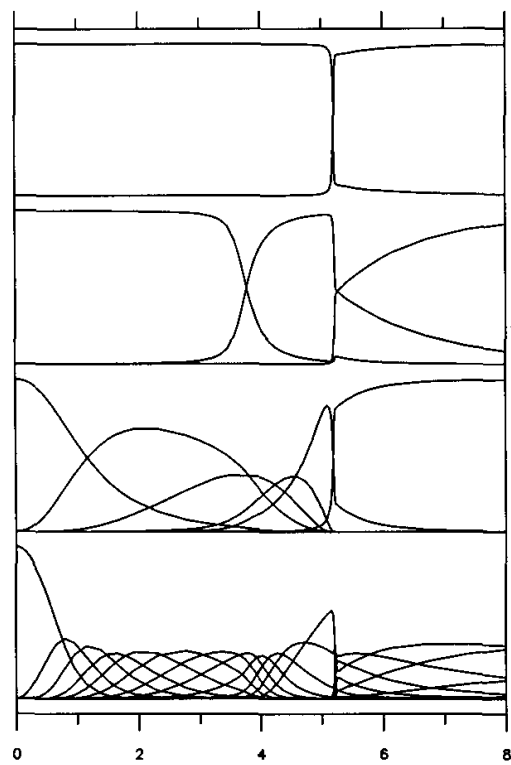

We can easily spot the presence of accretion onto a white dwarf. As shown by the formulae for $T^{b l}$, small values of $R_{*}$ produce high radiation temperatures; for white dwarfs they easily reach $T^{b l} \approx 10^{6} \mathrm{~K}$. Fe is then ionized to $\mathrm{Fe}^{+9}$ and higher, and we expect $[\mathrm{Fe}$ $\mathrm{X}$ ] $\lambda 6374$ or [Fe XIV] $\lambda 5303$ to be prominent lines in the spectra. However, neither [Fe X] nor [Fe XIV] are prominent in symbiotic spectra. In agreement with Kenyon and Webbink (1984), who for different reasons arrive at the same conclusion, we can probably rule out accretion on white dwarfs as relevant for symbiotic systems.

In Figure 7 we show the fractional ionizations resulting from the radiation field of an accreting white dwarf with $M=1 M_{\odot}, R^{*}=0.009 R_{\odot}$, and an accretion rate of $10^{-6} M_{\odot} / y r$. 
Within the disc we find $T_{\max }(d i s c)=250000 \mathrm{~K}$. The ionizing field is dominated by the boundary layer with $T=1.2 \cdot 10^{6} \mathrm{~K}$. The ionization structure is qualitatively different from those shown in Fig. 3 - 5. Helium does not completely recombine at the border of the $\mathrm{H}^{+}$-zone, nor does $\mathrm{Fe}$. This behaviour has the following reason: The combined disc and boundary-layer continuum is relatively flat, with an important fraction of hard photons having energies far beyond the $\mathrm{He}^{+}$ionization limit. Even in the $\mathrm{He}^{+}{ }_{-}$-zone, there is still a sufficient quantity of hard photons to keep a few $\%$ of He doubly ionized. When the bulk of the photons capable of easily ionizing $\mathrm{H}$ have disappeared, hydrogen will become mainly neutral. But with the disappearance of about $90 \%$ of the $e^{-}$, recombination for other elements is more difficult. Thus, the remaining hard photons, peaking in our case at about $10 \AA$, are able to keep He and other elements ionized. We thus have an outer part of the nebula, where hydrogen is neutral but many other elements are partially ionized.

\section{Outlook}

In recent years the models have evolved away from full spherical symmetry in radiation and mass-loss. For the interpretation of radio observations Taylor and Seaquist (1984) have modelled the symbiotic nebula as consisting of hydrogen only. They also simplified the treatment of ionization and recombination. Thus, they are able to calculate analytically the extent of the ionization region. Their basic model is the same as the one shown in Figure 2. By comparing the analytical results with much more extended model calculations, Nussbaumer and Vogel (1987, this publication also contains corrections to the formulae given by Taylor and Seaquist) show that the analytical treatment can serve as a very useful first approximation.

To account for the observed X-ray emission from HM Sge, Willson et al.(1984) propose a model, where the head-on collision of two stellar winds is supposed to produce a collisionally ionized hot region as a source of free-free radiation. However, the full implications for line and continuum spectra have not been calculated. But, as they need only $\approx 10^{-11} M_{\odot}$ to explain the X-ray emission, which is a tiny fraction of the total nebula, we obviously deal with fringe effects - this remark does not imply an invitation to disregard fringe-happenings. $\mathrm{X}$-ray emission has also been detected from the jet of $\mathrm{R}$ Aquari. In connection with the model proposed by Kafatos et al.(1986), Viotti et al.(1987) feel that the observed $\mathrm{X}$-ray emission of $\mathrm{R}$ Aqr may originate either in a radiatively or a collisionally ionized part of the jet. We see, that in spite of the fact that the normal spectrum of symbiotic stars indicates radiative ionization, collisional ionization may after all be active in some parts.

I now want to trace the steps the ionization models have followed, or are likely to follow. (I design the center of mass-loss as $C_{w}$ and the origin of the ionizing flux as $C_{r}$.) Published results:

1) Spherically symmetrical radiation field centered on $C_{r}$, spherically symmetrical mass-loss centered on $C_{w}$. The two centers coincide. The ionized nebula is spherically symmetrical around $C_{r}=C_{w}$. The computational problem is one-dimensional. Often this approximation is further simplified to a one-point model for the nebula.

2) Spherically symmetrical radiation field, spherically symmetrical mass-loss. The two centers $C_{r}$ and $C_{w}$ do not coincide. The resulting ionzed nebula is axially symmetrical around the line $C_{r}-C_{w}$. The computational problem is two-dimensional. 
Still to be done:

3) Disclike radiation field, spherically symmetrical mass-loss. The two centers $C_{r}$ and $C_{w}$ do not coincide. The only symmetry of the resulting ionzed nebula is relative to the plane containing the disc, and to the plane at a right angle, containing the line $C_{r}$ $-C_{w}$. The computational problem is three-dimensional with a certain facilitation due to the properties of symmetry

4) Disclike radiation field, mass-loss through one point. This corresponds to an accretion disc as radiation source and mass-loss via Roche-lobe overflow.

5) And further. In order to copy nature perfectly, we would certainly have to abandon any symmetry in mass-loss and radiation field.

We have seen that it is rather difficult to distinguish on purely spectroscopical grounds between blackbody radiation sources and accretion disc radiation sources. We also have to ask, how far we want to push modelling, so as not to introduce many more free parameters than can be fixed by observations.

In the ionization models employed to calculate symbiotic systems optical depth effects are usually taken into account only in a first approximation (see section 4.1). These methods will have to be extended to account properly for radiative transfer. Although it is useful to pay attention to what is done in other fields of astrophysics, where the nebular spectrum also contains major clues about the physical environment, we must always take into account the particular situation of symbiotic nebulae. Thus, in comparison with planetary nebulae, our nebular densities are higher by several powers of ten.

Up to now I have not mentioned the presence of dust. On this point I should like to point to Allens' (1982) comments. He considers the presence of dust as certain for D-type symbiotics, with some dust present in S-type symbiotics. Dust found in the interstellar medium is thought to be created in the winds of red giants and supergiants. But as shown by observations of planetary nebulae, dust is not necessarily the same everywhere. - A review of work up to 1982 on dust in planetary nebulae has been given by Barlow(1983). - The presence of dust, and whether observationally determined element abundances in symbiotic stars are influenced by condensation into dust grains, is in itself of high interest. In the model adopted, the symbiotic nebula is linked directly to the wind of the red giant. At a distance of $2 \cdot 10^{13} \mathrm{~cm}$ (see Fig. $3-5$ ) the not attenuated radiation field of a $T^{*}=150000 \mathrm{~K}$, $R^{*}=9 \cdot 10^{8} \mathrm{~cm}$ star would result in dust temperature of $\approx 700 \mathrm{~K}$. At the same distance a giant with $T^{*}=3000 \mathrm{~K}, R^{*}=7 \cdot 10^{12} \mathrm{~cm}$ gives $T^{d u s t}=1250 \mathrm{~K}$, if calculated on the basis of energy balance. To deal properly with dust, its presence will have to be included in the ionization model. There it will also have to be considered as an agent altering the ionizing radiation field.

As concluding remark I want to stress the importance of backing up speculative ideas about symbiotic systems, by matching them against properly calculated line and continuum fluxes. This requires ionization models, which abandon many of the simplifications employed up to now.

Acknowledgments: I thank H.M. Schmid and M. Vogel for discussions and help. 


\section{References:}

Allen, D.A.: 1982, The Nature of Symbiotic Stars, IAU Coll. No.30, ed M. Friedjung and R. Viotti, D. Reidel Dordrecht, p.27

Barlow, M.J.: 1983, IUE Symp.103, Planetary Nebulae, ed. D.R. Flower, p 105, Reidel (Dordrecht)

Feast, M.W., Whitelock, P.A., Catchpole R.M., Roberts, G., Carter, B.S.: 1983, Monthly Notices Roy.Astron.Soc. 202, 951

Hayes, M.A., Nussbaumer, H.: 1986, Astron.Astrophys. 161, 287

Kafatos, M., Michalitsianos, A.G., Hollis, J.M.: 1986, Astrophys.J.Suppl. 62, 853

Kenyon, S.J., Webbink, R.F.: 1984, Astrophys.J. 279, 252

Livio, M., Soker, N., de Kool, M., Savonije, G.J.: 1986, Monthly Notices Roy.Astron.Soc. 222, 235

Lynden-Bell, D., Pringle, J.E.: 1974, Monthly Notices Roy.Astron.Soc. 168, 603

Nussbaumer, H., Schild, H.: 1979, Astron.Astrophys. 75, L19

Nussbaumer, H., Schild, H.: 1981, Astron.Astrophys. 101, 118

Nussbaumer, H., Schild, H., Schmid, H.M., Vogel, M.: 1987, Astron.Astrophys. (to be submitted)

Nussbaumer, H., Schmutz, W.: 1983, Astron.Astrophys. 126, 59

Nussbaumer, H., Stencel, R.E.: 1987, Exploring the Universe with IUE, ed. Y. Kondo et al., Reidel Dordrecht, p.203

Nussbaumer, H., Vogel, M.: 1987, Astron.Astrophys. 182, 51

Paczynski, B., Rudak, B.: 1980, Astron.Astrophys. 82, 349

Penston, M.V., Benvenuti, P., Cassatella, A., Heck, A., Selvelli, P., Machetto, F., Ponz, D., Jordan, C., Cramer, N., Rufener, F., Manfroid, J.: 1983, Monthly Notices Roy.Astron.Soc. 202,833

Pringle, J.E.: 1977, Monthly Notices Roy.Astron.Soc. 178, 195

Pringle, J.E.: 1981, Ann.Rev.Astron.Astrophys. 19, 137

Regev, O.: 1983, Astron.Astrophys. 126, 146

Shakura, N.I., Sunyaev, R.A.: 1973, Astron.Astrophys. 24, 337

Taylor, A.R., Seaquist, E.R.: 1984, Astrophys.J. 286, 263

Viotti, R., Piro, L., Friedjung, M., Cassatella, A.: 1984, Astrophys.J. 319, L7

Vogel, M.: 1988, this volume

Willson, L.A., Wallerstein, G., Brugel, E.W., Stencel, R.E.: 1984, Astron.Astrophys. 133, 154 